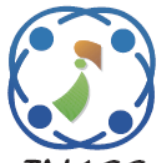

\title{
Elaboration of Psychological Ontology that Integrates the Problem of Human Frailty in COVID-19 Context
}

\author{
Lamia Moudoubah $^{1 *} \quad$ Kamal El Guemmat $^{1} \quad$ Khalifa Mansouri $^{1} \quad$ Mohammed Qbadou $^{1}$ \\ ${ }^{I}$ Signals, Distributed Systems and Artificial Intelligence Laboratory, ENSET Mohammedia, \\ University Hassan II, Casablanca, Morocco \\ * Corresponding author's Email: lamiae.modobah@gmail.com
}

\begin{abstract}
In terms of mental health, COVID-19 has a great impact on people's psychology. It can cause a certain degree of fear, worry, boredom, depression, eating disorders, loneliness and sometimes self-aggression or suicidal behaviour. The purpose of this article is to explore the psychological consequences of health problems related to COVID-19. During this period, some literature appears to analyze the psychological behaviour of individuals. However, in the sense of domain ontology, there is a lack of knowledge about modelling specific knowledge in the field of psychology and to have formal semantics. Through this article, we present a psychology-based method to analyze human behaviour and frailty during COVID-19. We chose IR (Information Research) based on TOR (Termino-Ontology Resources) as the method to construct the resulting ontology modelling the state of mental health in a COVID-19 context, an ontology that avoids ambiguities in the concepts that model the domain in question. The result of our research is the ontology described in Fig. 5, 6, 7, 8 and 9. The concepts that make up our ontology are derived from the results of the calculation of scores through Eq. 6, which consists of calculating the Score (Cij), then the $\mathrm{CI}(\mathrm{c})$ through the calculation of $\mathrm{P}(\mathrm{c})$ which is 0.18 in the term ti= anxiety, and therefore $(-\log (0.25))$ for the calculation of $\mathrm{CI}(\mathrm{c})$. These results concern the term "anxiety" in our ontology, following the same logic for the other terms. The lack of a psychological ontology modelling the concepts, those correspond to the fragility of people's mental health during the COVID-19 pandemic, so we thought about developing an ontology that would fill this gap.
\end{abstract}

Keywords: COVID-19, Psychology, Domain ontology, Disambiguation, SKOS.

\section{Introduction}

There is no doubt that the COVID-19 pandemic will remain engraved in the memory of everyone, regardless of their country, lifestyle or socioeconomic status, they will all be affected by this crisis. Psychologically, the confinement situation has brought us a new way of life, and its impact is significant. Anxiety, fear, depression, irritability, anger, confusion, social isolation, drug addiction, or drug-related behaviours, insomnia, aggressive behaviour and violence, etc. are generally the psychological and behavioural consequences of midlife. This unique environment can sometimes reveal the worst of human nature.

In this unprecedented situation everyone is asking a lot of questions. For example: how will our work be affected? What is the current psychological state of the family members of the deceased who were buried anonymously? What will happen tomorrow? What are the consequences for our mental health? Etc. In fact, not everyone has the same ability to adapt to this situation. The COVID-19 epidemic has made visible the weaknesses in the mental health of the general population, whether or not they suffer from psychiatric illnesses.

Several works have addressed mental health during the pandemic in the year 2020. [1] Have saying that "Coronavirus is a big challenge for a human being. People must be given a deep and thorough understanding of the deadly pandemic. They must be prepared for their current as well as prolonged post-pandemic effects on their lives and livings. Decisions of some countries are, to some extent, in the right direction. Restricted and extended 
lock down is not the solution to this drastic scenario because it has proved to be economically disastrous. The revival of social and economic activities is undeniable. Still, along with that, people should be aware of how they need to play their part and keep the balance in their outdoor activities and life-saving measures to protect themselves and their community. In that respect, the media and governing bodies are bound to do their duties honestly and vigilantly in the welfare of human beings and humanities."

According to [2], "COVID-19 has had a profound effect on our thoughts, emotions, and behavior. Understandably, it has generated much fear and panic globally. Mental health correlates to the pandemic have been widespread and varied in presentation, related to vulnerability and pre-morbid functioning, with social and economic factors contributing heavily. Commenting on national survey data during COVID19, the United Nations (2020) raised concern after studies showed distress prevalence ranging from $35 \%$ to $60 \%$. A recent population-based survey found that $54 \%$ of South Africans viewed themselves as moderate to high risk for contracting the virus, suggesting some level of anxiety about their current situation [3]. Another community survey of over 12,000 respondents noted significant negative emotions including $33 \%$ of adults depressed and $45 \%$ fearful, with fear, stress, and depression more prevalent in those fewer than 40 years of age than older people [4]. More worrying, however, is that the increased mental health service needs are surfacing during a time of reduced mental health service provision due to the risks associated with in-person consultations. In attempts to limit the spread of infection, large numbers of mental health service providers have opted to offer virtual consultations. The extent to which, this mode of consultation is meeting the needs of all concerned in the current situation, is yet to be determined, considering the diversity of those in need, especially in terms of age, educational level, technological sophistication, financial resources and numerous other variables - all an indication of just how egalitarian the virus is."

According To [26] "Beyond behaviour change, health psychology also has a role in understanding how people might respond to and cope with the threat of a global pandemic and changes to their lives that are made in an effort to reduce that threat".

Through the articles of the authors cited in the two preceding paragraphs, we have been able to deduce that the research carried out in the context of covid-19 is rich in detailed information on psychology and mental health.

At the level of ontological modeling, several works have been developed in the Covid-19 period.
These works concern the modeling of data related to Covid-19 for the valorization and the improvement of the statistics as in [27]. As well as work on the development of ontology related to the analysis of disease data in the context of Covid-19 such in [28].

Cependant, il y'a un vrai manque au niveau de la modélisation de l'ontologie intégrante du problème de la fragilité de la santé mentale des personnes pendant la pandémie. C'est pourquoi notre travail consistera à créer une ontologie qui sera la base d'une application de soutien psychologique qui sera réalisée par les auteurs de ce document. Therefore, the acquisition of knowledge in the field of psychology related to the COVID-19 pandemic is always reserved for individual users (researchers, psychiatry students, health professionals, people with COVID-19, family members of people with COVID19, teachers, parents, educators, etc.). Therefore, there is a need for a more powerful search tool that allows users to easily and quickly find relevant information that meets their needs. Our research will focus on the creation of a psychological ontology based on IR (information retrieval) "OntoPsyCOVID-19" based on the reuse of TOR (TerminoOntological Resources).

Nevertheless, although many semantic resources have been developed in the medical field, there is still a need to cover specific areas such as the psychological field, where knowledge of the field is regularly updated. Access to knowledge in this field remains an important issue for researchers and practitioners today. Such modeling can be done through domain ontology [5]. However, it should be noted that the construction of domain ontology remains a fastidious and costly task, which requires an elaborate methodology to lighten the process. No standard approach exists as it is the case in the field of databases [6].

To build the proposed ontology, we chose to work with the method of ontology acquisition from texts and TOR, by proceeding as follows; First, we were able to extract concepts from documents (texts, articles, ontology, books and dictionaries) automatically using a concordance and using queries to which our ontology will respond. We were interested in identifying representative concepts in textual corpora, in their disambiguation, in their weighting, and then in identifying the relations between these concepts.

To justify our choice for the above-mentioned method, we are obliged to point out that few research groups propose methodologies for the construction of ontology. However, ontological engineering consists in the study of methods; each working group uses its own methodology [6]. In this paragraph, we have 
based ourselves on the article in [29] to present the most important methodologies for ontology construction. From the previous work, we were able to extract four main categories of approaches: 1approaches for building ontology from scratch, 2approaches for building ontology from texts, 3approaches based on the reuse of existing TORs, 4approaches based on crowd sourcing. The choice of one methodology or another is based on the use we want to make of our ontology and also on the resources available for the construction of our ontology.

Approach 1 is a manual approach used by ontology experts, in the work of [30] and [31]. Then, more detailed methodologies such as Meth-ontology [29] and the more recent work of Sure and colleagues [32] appeared [6]. In these methodologies, the sources of knowledge are generally constructed by humans, including experts in the field of application [6]. Specific knowledge acquisition techniques (brainstorming meetings, interviews with experts, etc.) are necessary, but note that there is no guarantee that the knowledge described is exhaustive [6]. Approach 2 is an approach very often used in recent years, the disadvantage of this approach lies in the corpus of text used, since it forms the basis of ontology construction, it must therefore be rich and tied together. The analysis and processing of textual documents thus becomes simpler [6]. What motivates the development of methodologies for ontology construction from texts is that texts are considered to be good sources of knowledge. These approaches often combine linguistic, statistical and/or machine learning techniques to extract ontological knowledge from texts [33]. Depending on our resources, we have adopted this second approach combined with approach 3, which is the approach based on the reuse of existing TOR. To simplify the task of constructing the proposed ontology, we advocated the reuse of existing TORs [34]. These approaches focus on the exploitation of all or part of the information contained in these often informal sources for the development of new ontology or the enrichment of existing ontology [6]. They often seek to capture the implicit knowledge contained in these resources, generally represented in informal languages [35], to describe them in a formal model [6] and this is the objective of this paper. We cannot close this section without mentioning the crowd sourcing approach, which is a complementary approach to classical construction methodologies by allowing lightening certain tasks [6].

They exist Several methods of semantic information retrieval are generally based on keyword representation, also called word-bag representation; documents and queries are described by a set of words (or even n-grams) they contain; the correspondence between a document and a query is based on the number of words they share [6]. In addition to disregarding the order of words (technical communication vs. communication technique) and relying on a purely lexical matching (1:1 matching between words), they are thus affected by two main limitations: ambiguity (polysemy and homonymy) of words and synonymy. A term can be ambiguous (have several meanings) and its meaning depends in this case on its context of use [6].

Many are the statistical techniques most commonly used in IR to overcome the limitations related to term ambiguity and synonymy, such as LSA which uses algebraic techniques based on the frequency and co-occurrence of words in documents to construct "concepts". The advantage of this approach is that it does not require external knowledge. Consequently, it remains independent and applicable in any field. Moreover, associations between terms (synonymy, polysemy) are partially taken into account, but it remains limited for the treatment of large collections of documents since it depends on the SVD which is computationally heavy. In addition, updating indexes is difficult when new documents are added.

The approach of disambiguation that we adopt in this paper is inspired by the work of [14]. The advantage of this approach lies in its principle which consists, for each ambiguous term, in retrieving all the concepts it denotes and in finding the concept closest semantically to the other concepts in its context [6]. According to [8] "To estimate this proximity, some methods rely on semantic relations extracted from a knowledge source while others rely on more advanced similarity computation techniques". The disambiguation approach we use is inspired by the work of [8] and we have integrated a weight to measure the importance (for disambiguation) of each term in the context of the ambiguous term. The methodology used in this paper is described in more detail in Section 2.

The major argument justifying the choice of the SKOS language for the formalization of the proposed ontology is that SKOS is a data model intended to support TORs. And since we base our ontology construction on TORs, we are consequently required to adopt the SKOS language.

In the remainder of this document, the research methodology used is first presented in section II, then the construction of our OntoPsy-COVID-19 ontology in section III, followed by the formalization of the ontology in section IV. Then, presentation of the 
results and discussion in section V. Finally, conclusion in section VI.

\section{Research methodology}

\subsection{Why an ontology?}

Ontology have today a major place in the representation and modeling of knowledge [6]. They are used to formalize the knowledge of a domain and add a semantic layer to computer systems and applications [6]. Ontology is used to explicitly represent domain knowledge using a formal language so that it can be automatically manipulated and easily shared [6]. They are widely used in various research areas such as knowledge representation, IR [8] and data integration [12].

The notion of ontology has been redefined in the course of the debates it has been the subject of. We will retain the following definition [16]: "ontology is a standardized specification representing the classes of objects recognized as existing in a domain." While having a meaning for humans, computers must be able to use ontology to define the concept of domain in a standardized and consensual way, while meeting the needs of expected applications. The role of ontology in information retrieval lies in the link between knowledge and its expression in language: it must not only support research work, but also define resource files for annotation metadata. Some works such as [16] mention the need to associate independent dictionaries with ontology to mark concepts and relationships, all of which form ontology with lexical components.

\subsection{Type of ontology?}

There are different typologies that have been proposed to distinguish the multiple existing ontology: according to their coverage, according to their granularity or according to their level of formalisation. At the same time, the craze generated by the multiple functionalities offered by ontology has resulted in a very rapid development of "derived" resources that do not exactly comply with the definition and mode of use of ontology, particularly in the medical field [17].

Proposed ontology "OntoPsy-COVID-19" is a domain ontology, which allows expressing the knowledge of a specific "Psychological Domain", and it can be reused through applications in this domain. It is the most common type of ontology in ontology engineering [6].

\subsection{Methodology for the construction of 'OntoPsy-COVID-19'}

The method used to build the proposed ontology is IR (information retrieval) based on the reuse of TOR (Termino-Ontological Resources) [11]. TOR reuse approach: This approach focuses on the exploitation of all or part of the information contained in these often informal sources for the development of new ontology or the enrichment of existing ones. They often seek to capture the implicit knowledge contained in these resources, usually represented in informal languages [14] describe it in a formal model.

\subsubsection{The construction of document base}

In this part, we use the IR method based on domain ontology using the knowledge contained in semantic resources. In order to be able to answer the various questions that constitute the field of "psychology" related to the COVID-19 pandemic.

\subsubsection{Extraction of candidate terms}

All collected documents that form the basis of the text are processed and analyzed using the "AntConc" concordance. This tool allows users to make queries in the form of regular expressions in the text database and displays all databases containing the expression Part, to enrich the body during inspection.

\subsubsection{Disambiguation of terms}

This step allows, for each ambiguous term, to find the adequate concept corresponding to its context of use [6]. The approach of disambiguation with which we have worked is inspired by the work of [13] and we have integrated a weight allowing us to measure the importance (for disambiguation) of each term in the context of the ambiguous term. The principle of this method is, for each ambiguous term, to retrieve all the concepts it denotes and to find the concept that is semantically closest to the other concepts of its context [6]. This context is defined as an arbitrarily fixed text window and can be a sentence, a paragraph or even the whole document [6]. Thus, for each concept associated with the ambiguous term, its similarity score is estimated as the sum of the degree of similarity of this concept with each of the other concepts in its context [6]. For any other ambiguous term in the context, only its associated concept closest semantically to the target concept (i.e., having the highest degree of similarity with the latter) is considered instead of all the concepts it denotes [6]. At the end of this computation, the concept with the highest score is retained and assigned to the term.

The formula used by McInnes and Pedersen is described in more detail in [14]. The similarity between a concept denoted by a term to be 
disambiguated and the other concepts in its context is weighted by a coefficient indicating the importance of their associated terms in the disambiguation [14]. This weight, for a given term, is defined as the inverse of the number of concepts associated with it [14]. We consider that the less polysemic a term in the context, the more important it is for the disambiguation of ambiguous terms. Thus, for unambiguous terms, this weight is maximal $\left(w\left(t_{i}\right)=1\right)$.

Formally, $\mathrm{F}$ is the context of an ambiguous term $\mathrm{t}_{\mathrm{i}}$ associated with n concepts $C_{i}=\left\{C_{i l}, \ldots, C_{i n}\right\}$ and $\mathrm{T}$ is the set of terms of $\mathrm{F}$ extracted using the concordance.

The score of each $\mathrm{C}_{\mathrm{ij}}$ concept with $J \in\{1, \ldots, n\}$ is defined by the following equation:

$$
\begin{aligned}
& \operatorname{Score}\left(c_{i j}\right) \\
& =\sum_{\substack{k=1 \\
k \neq i}} \max _{\substack{|T| \\
w(t i)}} l \in[1, \ldots,|C K|] \quad\left(\operatorname{Sim}_{\text {Lin }}\left(c_{i j}, c_{k l}\right)\right)
\end{aligned}
$$

$\operatorname{Sim}_{L i n}\left(C_{i j}, C_{k l}\right)=$ the semantic similarity between the concepts $C_{\mathrm{ij}}$ and $\mathrm{C}_{\mathrm{kl}}$ defined in [14].

$W(t i)=$ a weight to indicate the importance of the concepts associated with the term $\boldsymbol{t}_{i}$ in disambiguation, it is calculated by :

$$
w(t i)=\frac{1}{\text { nbconcept }(t i)}
$$

Such that $\boldsymbol{n b c o n c e p t}\left(\boldsymbol{t}_{i}\right)=$ the number of concepts associated with $\boldsymbol{t}_{\boldsymbol{i}}$.

In this way, we have developed a clear set of concepts that can be used to index the processed documents.

\section{Construction of proposed ontology "OntoPsy-COVID-19"}

In this section, we build the ONTOPSY-COVID19 ontology in a clear, efficient and relevant way. As mentioned in the introduction of this article, our ontology will be based on information retrieval (IR) based on the reuse of TOR. The principle of IR is based on the following steps: 1) Users express their needs through requests (free text or forms). 2) The request is analyzed, processed and represented by a series of concepts in the documents that form the basis of our text.

Mental health information for the public can have a huge psychological impact. However, there are still some gaps in the strategies used to convey this information and the public does not benefit from psychological knowledge. The conceptual accessibility of information is the ability to find, understand and interpret psychological information in the context of COVID-19. People want to understand the mental state related to COVID-19, and they seek information about the mental health of people with the same experience and symptoms, as their behaviour may be similar to that of others in some cases. However, the mass dissemination of this information on the Internet may satisfy the needs of a few people.

\subsection{Starting postulate}

The initial hypothesis of text selection is knowledge, and therefore the concept of human frailty can be simulated in a covid-19 context, included in the text representing the field. As proof, Bachimont's work [16] adopted this point of view: "The work of modeling must be extracted from documents proven in practice and gathered in a corpus" [16].

Our choice of text is based on the following assumptions:- Since our aim was to highlight aspects of the functioning of terms in the field of psychology in relation to the COVID-19 pandemic, this choice of text should also be a choice of specialized texts, using terms. We consider the text as the material substrate carrying both semantic and pragmatic content. - The use of the proposed ontology should improve the understanding of the psychological state of people during the Covid-19 pandemic by a more efficient management of the information and documentary resources of the domain.

\subsection{The construction of the text base}

We used two types of documents obtained from the Internet: documents on the psychological field and the mental health of individuals related to COVID-19, and other documents related to general psychology.

Sources of documents used: According to [18], useful sources should have three properties: they represent related fields, they contain current information, and finally the relationship with the associated fields should be clear.

Depending on the quality described in the previous paragraph, the website or web page used to extract the documents that make up our text database is selected. For COVID-19, Google is used as a search engine to generate documents related to psychology. Several queries were launched to retrieve the desired documents, For documents concerning psychology in a covid-19 context; - 
Table 1. Text features

\begin{tabular}{|l|c|}
\hline $\begin{array}{c}\text { Texts by researchers } \\
\text { in the field of psychology } \\
\text { related to the pandemic } \\
\text { COVID-19 }\end{array}$ & $\begin{array}{c}\text { Texts related to the } \\
\text { field of psychology }\end{array}$ \\
\hline $\begin{array}{c}\text { Number of } \\
\text { documents }=85 \\
\text { Number of words }= \\
982868\end{array}$ & $\begin{array}{c}\text { Number } \\
\text { documents =15 } \\
\text { Number of words = } \\
491434\end{array}$ \\
\hline
\end{tabular}

Mental health related to the COVID-19 pandemic. Impact of COVID-19 on the mental health of individuals. -Pandemic and mental health. Psychological disorders related to covid-19. -The psychology of individuals during the course of the corona virus. Concerning documents related to psychology; -Psychology. -The mental health of persons. - Ontology of psychology. -Dictionary of psychology.

The documents that appear at the top, i.e., the most frequently referenced and popular documents, always meet the following standards: representativeness of the field, target audience (public health or mental health professionals), author of the page (mental health professional or not). These standards allow us to collect and sort the most appropriate documents to provide the terms that make up our ontology.

The source of our collected documents is not only documents, but rather ontology of psychology such as the Oxford Dictionary of Psychology and many other dictionaries of psychology. We have collected 100 documents in ENGLISH.

Table 1 contains functionalities related to the two types of text related to our search (the numbers provided in the table below do not take into account lists of non-alphanumeric characters or empty words).

\subsection{Extraction of candidate terms}

The step of term extraction is according to [19] "the task of automatically detecting, from a textual base, lexical units that designate concepts in restricted thematic areas" [19].

After constructing the document (this is the starting hypothesis of ontology), we found that the extracted expressions and lists of terms contained a lot of noise. Therefore, we use the AntConc concordance to select and identify candidate terms specific to the field of psychology.

Once we have imported the files that make up our text into the concordance, we can extract a set of terms and a series of words from the documents that make up the text. We were able to collect the following candidate terms in table 2 :
Table 2. Candidate terms collected

\begin{tabular}{|c|c|c|}
\hline ID & Term & Frequency \\
\hline 1 & Addiction & 22 \\
\hline 2 & Aggressiveness & 109 \\
\hline 3 & Worry & 287 \\
\hline 4 & Background & 31 \\
\hline 5 & Anticipation & 37 \\
\hline 6 & Antidepressants & 19 \\
\hline 7 & Anxiety & 516 \\
\hline 8 & Abdominal Anxiety & 1 \\
\hline 9 & Increased anxiety & 4 \\
\hline 10 & Generalized anxiety & 3 \\
\hline 11 & Wellness & 442 \\
\hline 12 & character & 461 \\
\hline 13 & Containment & 825 \\
\hline 14 & COVID-19 & 3430 \\
\hline 15 & Depression & 297 \\
\hline 16 & Drugs & 56 \\
\hline 17 & Emotion & 133 \\
\hline 18 & emotional & 182 \\
\hline 19 & Psychosocial intervention & 4 \\
\hline 20 & Insulation & 47 \\
\hline 21 & Sick & 764 \\
\hline 22 & Mental illness & 15 \\
\hline 23 & Negativism & 10 \\
\hline 24 & Personality & 306 \\
\hline 25 & Patient & 892 \\
\hline 26 & Health Care Providers & 65 \\
\hline 27 & Psychoanalysts & 24 \\
\hline 28 & psychological & 1098 \\
\hline 29 & Psychologists & 120 \\
\hline 30 & Psychotraumatology & 21 \\
\hline 31 & Quality of life & 131 \\
\hline 32 & Reintegration & 4 \\
\hline 33 & Relaxation & 37 \\
\hline 34 & Resilience & 157 \\
\hline 35 & Mental Health & 1877 \\
\hline 36 & post-traumatic stress & 61 \\
\hline 37 & Suicide & 143 \\
\hline 38 & Tele-consultation & 27 \\
\hline 39 & Trouble & 109 \\
\hline 40 & Emotional Disorder & 2 \\
\hline 41 & Eating Disorder & 3 \\
\hline 42 & Person & 8 \\
\hline 43 & Bipolar disorder & 4 \\
\hline 44 & Acute Stress Disorder & 3 \\
\hline 45 & Eating Disorder & 4 \\
\hline 46 & Trouble sleeping & 4 \\
\hline 47 & Neurotic disorder & 2 \\
\hline 48 & Panic Disorder & 3 \\
\hline 49 & Psychic disorder & 8 \\
\hline
\end{tabular}

\subsection{Disambiguation of terms}

So we moved on to the concept disambiguation stage, in which we tried to find the right concept that corresponds to the COVID-19 context. After using the concordance, we were able to deduce the 
candidate terms, but there are still polysemic terms. This is why we resorted to the disambiguation technique based on the semantic similarity between the collected candidate terms. We consider that the less polysemic a term in the context is, the more important it is for the disambiguation of ambiguous terms.

We applied McInnes and Pedersen's formula as follows:

We take the case of the Formal context $\mathrm{F}=$ increased anxiety an ambiguous term $\mathrm{t} i=$ anxiety associated with $\mathrm{n}=4$ concepts $C i=\left\{C i_{1}=\right.$ Anxiété, $\mathrm{Ci}_{2}=$ Anxiété abdominales, $\mathrm{Ci}_{3}=$ anxiety accrue, $C i_{4}=$ generalized anxiety $\}$ and $\boldsymbol{T}=\mathbf{4 9}$ all the terms that appear in Table 1, collected by the concordance software used 'AntConc'.

To calculate the score for each concept $\boldsymbol{C}_{i j}$ with $\boldsymbol{J}$ $\epsilon\{1, \ldots, 4\}$ which is defined by the following equation :

$$
\begin{aligned}
& \text { Score }\left(c_{i j}\right) \\
& =\sum_{\substack{k=1 \\
|T|}} \max (1, \ldots,|C K|] \quad\left(\operatorname{Sim}_{\text {Lin }}\left(c_{i j}, c_{k l}\right)\right) \\
& \times w(t i)
\end{aligned}
$$

With $\operatorname{Sim}_{L i n}\left(\boldsymbol{C}_{i j}, \boldsymbol{C}_{\boldsymbol{k}}\right)=$ the semantic similarity between the concepts defined above $C_{i j}$ and concepts $C_{k l}$ Knowing that the $C_{k l}$ are the concepts that make up the $T$. So the $K=\{1, \ldots, 49\}$ and the $l \in\{1, \ldots, 49\}$.

And $\mathbf{W}\left(\mathbf{t}_{\mathbf{i}}\right)=$ a weight to indicate the importance of the concepts associated with the term $\boldsymbol{t}_{i}$ in disambiguation, it is calculated by :

$$
w(t i)=\frac{1}{\text { nbconcept }(t i)}
$$

In our case the weight $\mathrm{w}(\mathrm{ti})$ :

$$
w(t i)=\frac{1}{4}
$$

Such as $\boldsymbol{n b c o n c e p t}\left(\boldsymbol{t}_{i}\right)=$ the number of concepts associated with $\boldsymbol{t}_{i}=4$.

By replacing the values obtained in the equation to calculate the score $(\mathrm{Cij})$ :

$$
\begin{aligned}
& \text { Score }\left(c_{i j}\right) \\
& \begin{array}{l}
=\sum_{\substack{k=1 \\
k \neq i}}^{|49|} \max \frac{1}{4}^{-\ldots,|45|]}\left(\operatorname{Sim}_{\operatorname{Lin}}\left(c_{i j}, c_{k l}\right)\right) \\
\end{array}
\end{aligned}
$$

To have the value of the score, we still have to detect the value of $\operatorname{Sim}_{L i n}\left(\boldsymbol{C}_{i j}, \boldsymbol{C}_{k l}\right)$.

To study the similarity of the $\mathrm{C}_{\mathrm{ij}}$ of ti concepts with the $\mathrm{C}_{\mathrm{kl}}$ de $\mathrm{T}$ concepts, we have used again the Resnik method introduced by Resnik in 1995 in [29], this method is clarified in [27].

The notion of informational content (IC) was first introduced by Resnik in [20]. It uses both ontology and corpus [21]. The informational content of a concept reflects the relevance of a concept in the corpus by taking into account its specificity or generality [21]. A general concept is said to subsume a more specific concept [21]. The frequency of concepts in the corpus is calculated in order to find the information content [21].

This frequency groups together the frequency of appearance of the concept itself as well as the concepts it subsume [21]. The formula is as follows:

$$
C I(c)=-\log (P(c))
$$

With $P(c)=$ the probability of finding an instance of the concept $\mathrm{c}$. These probabilities are calculated by: frequency $(c) / N$ where $\mathrm{N}$ is the total number of concepts, according to Lin in [27].

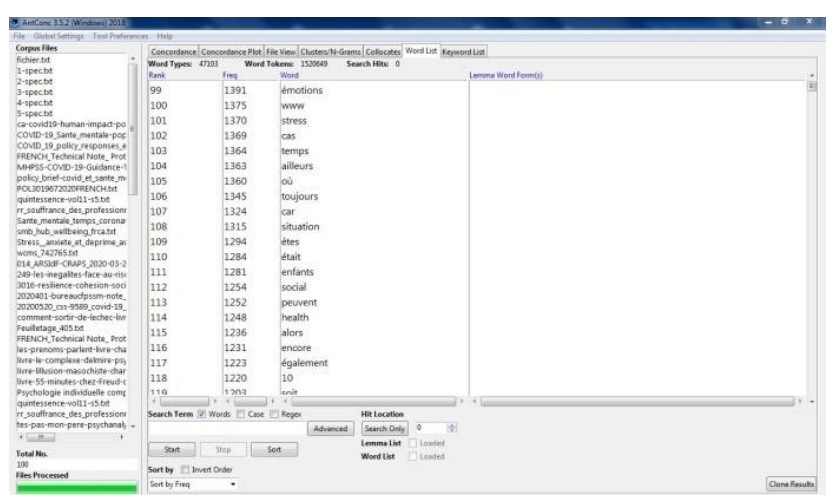

Figure. 1 Example of term search in the 'AntConc' concordance

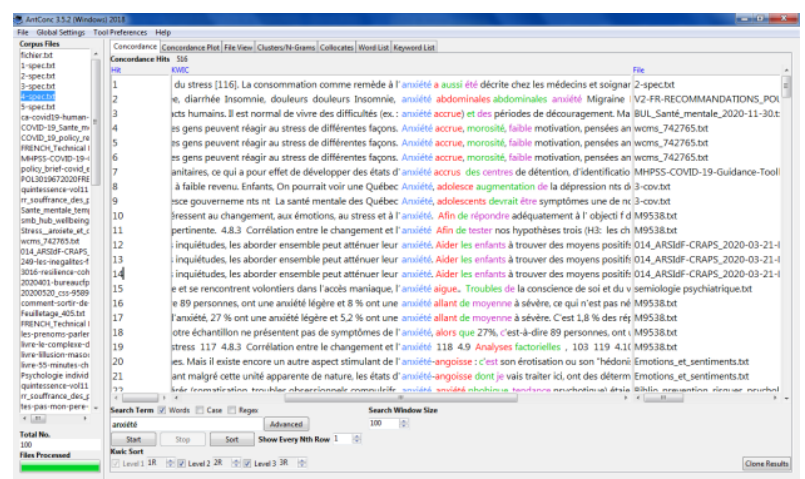

Figure. 1 Example of term search in the 'AntConc' concordance 
Table 3. Result of adopted Method

\begin{tabular}{|c|c|c|}
\hline Term & $\begin{array}{l}\text { Calcul du P(c) } \\
=(c) / N\end{array}$ & $\begin{array}{l}\text { Calcul } \\
\text { CI(c) } \\
=(- \\
\log (\mathbf{P}(\mathbf{c}))\end{array}$ \\
\hline Addiction & 1,18 & $-0,071$ \\
\hline Aggressiveness & 4,19 & $-0,62$ \\
\hline Anxiety & 0,18 & 0,74 \\
\hline Background & 1,19 & $-0,07$ \\
\hline Anticipation & 1,42 & $-0,15$ \\
\hline Antidepressants & $\mathbf{0 , 7 3}$ & $\mathbf{0 , 1 3}$ \\
\hline Wellness & 17 & $-1,23$ \\
\hline Character & 17,73 & $-1,24$ \\
\hline Containment & 31,73 & $-1,50$ \\
\hline COVID-19 & 131,42 & $-2,11$ \\
\hline Depression & 11,42 & $-1,05$ \\
\hline Emotion & 5,11 & $-0,70$ \\
\hline Patient & 34,30 & $-1,53$ \\
\hline Mental illness & $\mathbf{0 , 5 7}$ & 0,24 \\
\hline Personality & 11,76 & $-1,07$ \\
\hline Person & $\mathbf{0 , 3 0}$ & $\mathbf{0 , 5 2}$ \\
\hline Psychoanalysts & 0,92 & $\mathbf{0 , 0 3}$ \\
\hline psychological & 42,23 & $-0,62$ \\
\hline Psychologists & 4,61 & $-0,66$ \\
\hline Quality of life & 5,03 & $-0,70$ \\
\hline Relaxation & 1,42 & $-0,15$ \\
\hline Resilience & 6,03 & $-0,78$ \\
\hline Mental Health & 72,19 & $-1,85$ \\
\hline $\begin{array}{l}\text { post-traumatic } \\
\text { stress }\end{array}$ & 2,34 & $-0,36$ \\
\hline Suicide & 5,5 & $-0,74$ \\
\hline Tele-consultation & $\mathbf{1 , 0 3}$ & $-0,01$ \\
\hline Trouble & 4,19 & $-0,62$ \\
\hline
\end{tabular}

We have calculated the $P(c)=0.18$ in our case So:

$$
C I(c)=-\log (0.18)
$$

Resnik in [16] defines the semantic similarity between two concepts by the amount of information they share. This shared information is equal to the information content of the smallest generalizer (PPG) the most specific concept that subsume both concepts in ontology [21].

$$
\operatorname{Sim}(c 1, c 2)=C I(\operatorname{ppg}(c 1, c 2))
$$

We have therefore been able to deduce the formula for calculating the similarity $\operatorname{Sim}_{L i n}\left(C_{i j}, C_{k l}\right)$ :

Where:

$$
\operatorname{Sim}_{L i n}\left(C_{i j}, C_{k l}\right)=C I\left(p p g\left(C_{i j}, C_{k l}\right)\right)
$$

We then take the CI of the smallest generalize between the concepts, we thus obtain the value of
$\operatorname{SimLin}(\mathrm{Cij}, \mathrm{Ckl})$ and we replace it in our equation of calculation of the score for disambiguation. And the concept with the highest score is retained and assigned to the term.

Through this method, a set of unambiguous concepts is deduced. Each concept is defined by a term and usually an intention or extension in table 3 .

\section{Formalization of "OntoPsy-COVID-19"}

The final step is to use formal and expressive language to describe the ontology derived from the previous steps. For this, the SKOS reference language is used. SKOS (Simple Knowledge Organisation System) is a representation language of concept schemas, which covers documentaries languages such as thesauri, classifications, subject heading lists, taxonomies, folksonomies, etc [22]. Its name has been chosen to highlight the very objective of this language: to propose a system allowing the expression and management of machineinterpretable models in the semantic web perspective [22]. This model is defined as "simple" as opposed to other models, such as OWL (Ontologic Web Language), which are more capable of representing richer semantic structures such as ontology, but also more complex to use [22].

\subsection{Identification of relations between concepts}

To identify the relations between the concepts that make up our ontology, we have used the ACF formal concept analysis method, which consists of using the syntactic analysis of the sentences automatically obtained by the concordance to construct the formal context by removing the nouns and verbs from the analysis. The object in the formal context is the noun that appears as the direct subject or complement of the object. The attributes of the formal context are the verbs of the sentence in question, and they end with the suffix -ABLE when they come from the direct object complement (COD).

Definition of Formal Context: A formal context is a triplet

$$
K=(G, M, I)
$$

Where $\mathrm{G}$ is a set of objects, $\mathrm{M}$ is a set of attributes and $\mathrm{I}$ is a binary relation between $\mathrm{G}$ and $\mathrm{M}$ called $\mathrm{K}$ 's incidence relation and checking

$$
I \subseteq G \times M
$$

A pair

$$
(g, m) \in I
$$


(Also denoted gIm) means that the object

$$
g \in G
$$

Has the attribute [23].

$$
m \in M
$$

A formal context can be represented in the form of a table where the rows correspond to objects and the columns correspond to attributes [23]. The cells of the table are filled as follows: if the ith object $g$ is in relation I to the jth object, then the cell intersection of row $i$ and column $j$ contains " $\times$ ", otherwise the cell is empty [23].

By applying this method, we were able to deduce the formal concepts of our ontology in Table 4.

\begin{tabular}{|c|c|c|c|c|c|c|c|c|c|c|c|c|c|c|c|c|c|c|c|c|c|c|c|c|c|c|}
\hline & 1 & 2 & 3 & 4 & 5 & 6 & 7 & 8 & 9 & $\begin{array}{l}1 \\
0 \\
\end{array}$ & $\begin{array}{l}1 \\
1 \\
\end{array}$ & $\begin{array}{l}1 \\
2 \\
\end{array}$ & $\begin{array}{l}1 \\
3 \\
\end{array}$ & $\begin{array}{l}1 \\
4\end{array}$ & $\begin{array}{l}1 \\
5\end{array}$ & $\begin{array}{l}1 \\
6\end{array}$ & $\begin{array}{l}1 \\
7\end{array}$ & $\begin{array}{l}1 \\
8\end{array}$ & $\begin{array}{l}1 \\
9\end{array}$ & $\begin{array}{l}2 \\
0\end{array}$ & $\begin{array}{l}2 \\
1\end{array}$ & $\begin{array}{l}2 \\
2\end{array}$ & $\begin{array}{l}2 \\
3\end{array}$ & $\begin{array}{l}2 \\
4\end{array}$ & $\begin{array}{l}2 \\
5\end{array}$ & $\begin{array}{l}2 \\
6\end{array}$ \\
\hline Person & $x$ & & & & $x$ & & $x$ & & & & & & & & & & & & & & & & & & & \\
\hline Patient & $x$ & & & & & & $x$ & & $x$ & & & & $x$ & & & & & & & & & & & & & \\
\hline Covid-19 & & $x$ & & & & & & & & & & & & & & & $x$ & & & & & & & & & \\
\hline $\begin{array}{l}\text { Mental } \\
\text { Health }\end{array}$ & & & $x$ & & & & & & & & & & & & & & & & & & & & & & & \\
\hline Back & & & & $x$ & & & & & & & & & & & & & & & & & & & & & & \\
\hline $\begin{array}{c}\text { Personalit } \\
\mathrm{y}\end{array}$ & & & & & & $x$ & & & & & & & & & & & & & & & & & & & & \\
\hline $\begin{array}{l}\text { Psycholog } \\
\text { ists }\end{array}$ & & & & & & & & & & $x$ & $x$ & & & & & & & & $\times$ & & & & & & $\times$ & \\
\hline $\begin{array}{c}\text { Psychoana } \\
\text { lysts }\end{array}$ & & & & & & & & & & $x$ & $\times$ & & & & & & & & $\times$ & & & & & & $x$ & \\
\hline Trouble & & & & & & & & $x$ & & & & & & & $x$ & & $x$ & & & & & $x$ & & & & \\
\hline $\begin{array}{l}\text { Mental } \\
\text { illness }\end{array}$ & & & & & & & & $x$ & & & & & & & $x$ & & $x$ & & & & & $x$ & & & & \\
\hline Agony & & & & & & & & $x$ & & & & & & & $x$ & & & & & & & $x$ & & & & \\
\hline Anxiety & & & & & & & & $x$ & & & & & & & $x$ & & & & & & & $x$ & & & & \\
\hline $\begin{array}{c}\text { Aggressiv } \\
\text { eness }\end{array}$ & & & & & & & & $x$ & & & & & & & $x$ & & & & & & & $x$ & & & & \\
\hline Addiction & & & & & & & & $x$ & & & & & & & $x$ & & & & & & & & & & & \\
\hline $\begin{array}{c}\text { Depressio } \\
\mathrm{n}\end{array}$ & & & & & & & & $x$ & & & & & & & $x$ & & & & & & & $x$ & & & & \\
\hline $\begin{array}{c}\text { Antidepre } \\
\text { ssant }\end{array}$ & & & & & & & & & & & & $x$ & & & & & & & & & & & & & & \\
\hline
\end{tabular}

Table 4. Formal Context of the Proposed Ontology 


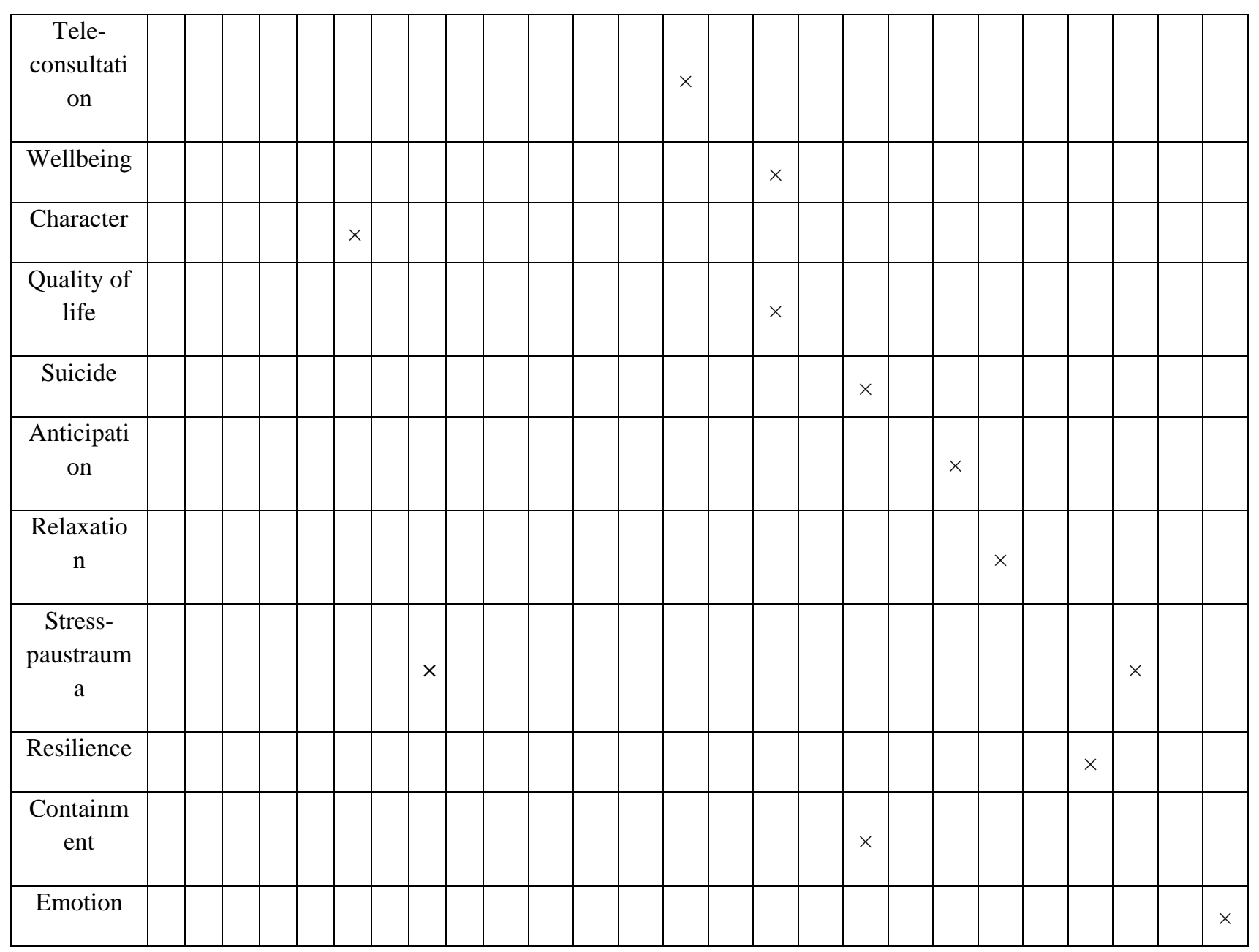

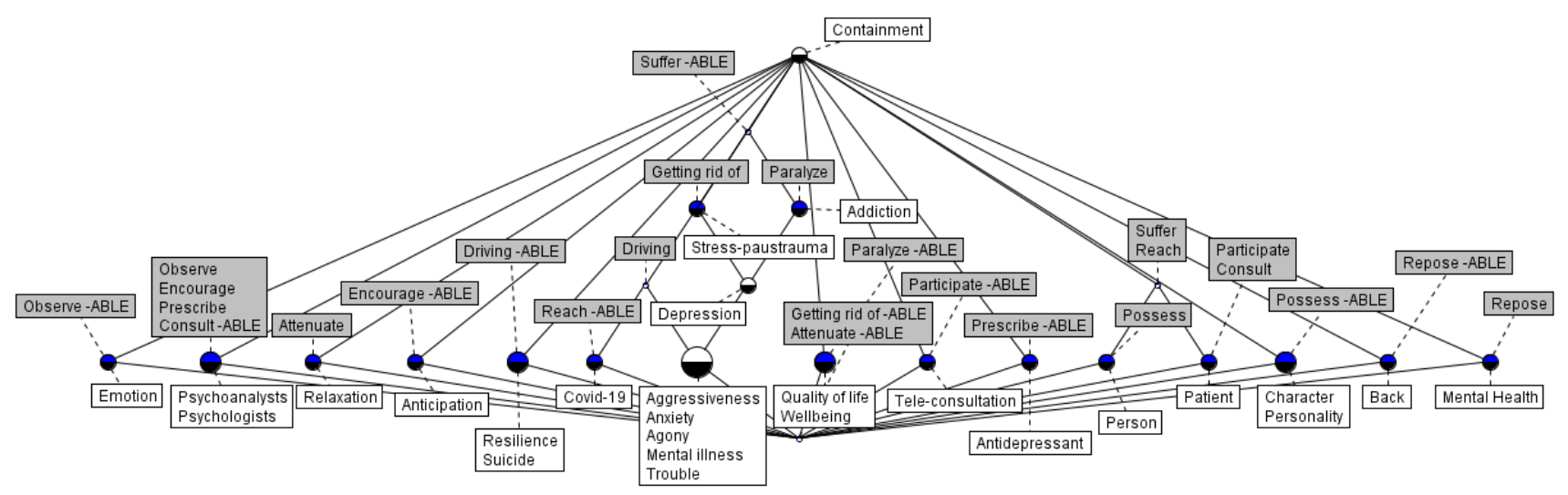

Figure. 4 OntoPsy-COVID-19 Conceptual Lattice for Ontological Interpretation

Identification of the numbers listed in Table 4:

1. Reach

2. Reach -ABLE

3. Repose

4. Repose-ABLE

5. Possess

6. Possess-ABLE

7. Suffer
8. Suffer-ABLE

9. Consult

10. Consult -ABLE

11. Prescribe

12. Prescribe-ABLE

13. Participate

14. Participate-ABLE

15. Paralyze 
16. Paralyze -ABLE

17. Driving

18. Driving -ABLE

19. Encourage

20. Encourage -ABLE

21. Attenuate

22. Attenuate -ABLE

23. Getting rid of

24. Getting rid of $-\mathrm{ABLE}$

25. Observe

26. Observe-ABLE

\subsection{Visualization of relations between concepts through the lattice}

The lattice of formal concepts can be represented visually in a Hasse diagram [24]. Each node of this diagram represents a formal concept; each arc represents a subsumption relation [24]. To improve the readability of the lattice it is possible to represent it in a reduced form, which consists in transferring the labels of the intentions to the most general concept possible in the lattice and the labels of the extensions to the most specific concept [24].

To trace the lattice that concerns our ontology, we chose to use ConExp as a tool that allowed us to trace the terminology to be interpreted by the SKOS in the following section. We chose ConExp because it offers a compact and modular representation of the lattice.

In order to use ConExp, we started by entering the above table in the 'context Editor' part, in order to conceptualize the latice line diagram through the calculation of the concepts (Fig. 5).

Concept formel «standard »

- Concept formel avec extension propre

Concept formel avec intension propre

Concept formel « blanc »

\subsection{Model SKOS}

Recommended by the W3C since August 2009, SKOS is a data model designed to support TORs, such as terminologies, thesaurus or taxonomies [25].

We have chosen to use SKOS in this article because we want to describe in detail the level of vocabulary that constitutes the concept of the proposed ontology "OntoPsy-COVID-19". SKOS provides a simple mechanism to support the representation. Structured vocabulary. Each concept is defined as an RDFS class with a set of attributes. A concept has a URI for its identification, a preferred term (or one per language in the case of multilingual resources), possible synonyms (or alternative terms) and one or more definitions. The term (or label) is a

string. These concepts are linked by generalization / specialization (skos:broader and skos:narrower) and by association (skos:related). Fig. 5, 6, 7, 8 and 9 shows the concepts of the proposed ontology 'OntoPsy-COVID-19' in SKOS.

\section{Result and Discussion}

The terminology and ontology levels are not differentiated. We are inspired by several works and in particular the ontology of psychology APAONTO, but we wish to go further in this study and manipulate this ontology model in SKOS through a psychological support application that will be realized soon.

Ontology assessment is always an open research question. As part of this work, the ontology is being verified in practice. Different aspects will be evaluated: the relevance of the ontology for the target application, its coverage in the field, etc. In fact, the generated ontology will soon be refined and verified by experts in the field, and will be evaluated in practical applications (in progress).

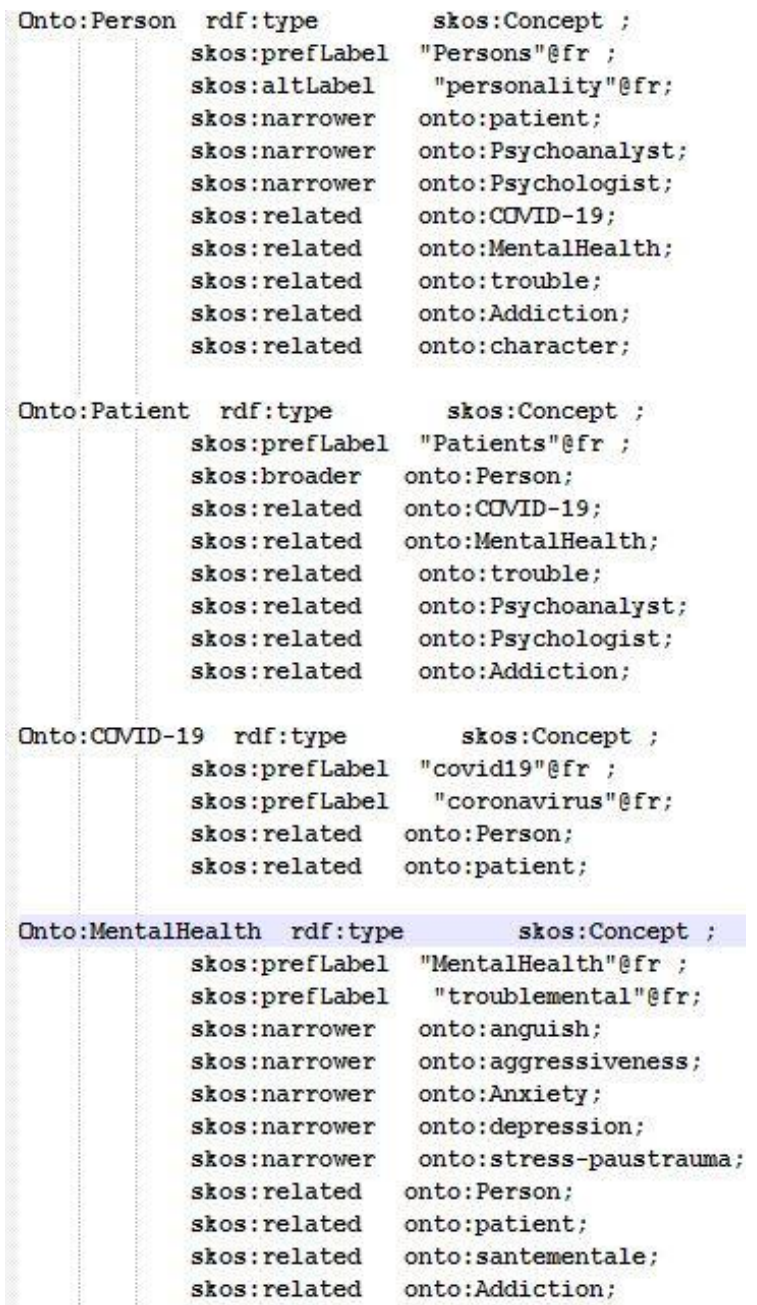

Figure. 5 Presentation of ontology 'OntoPsy-COVID- 


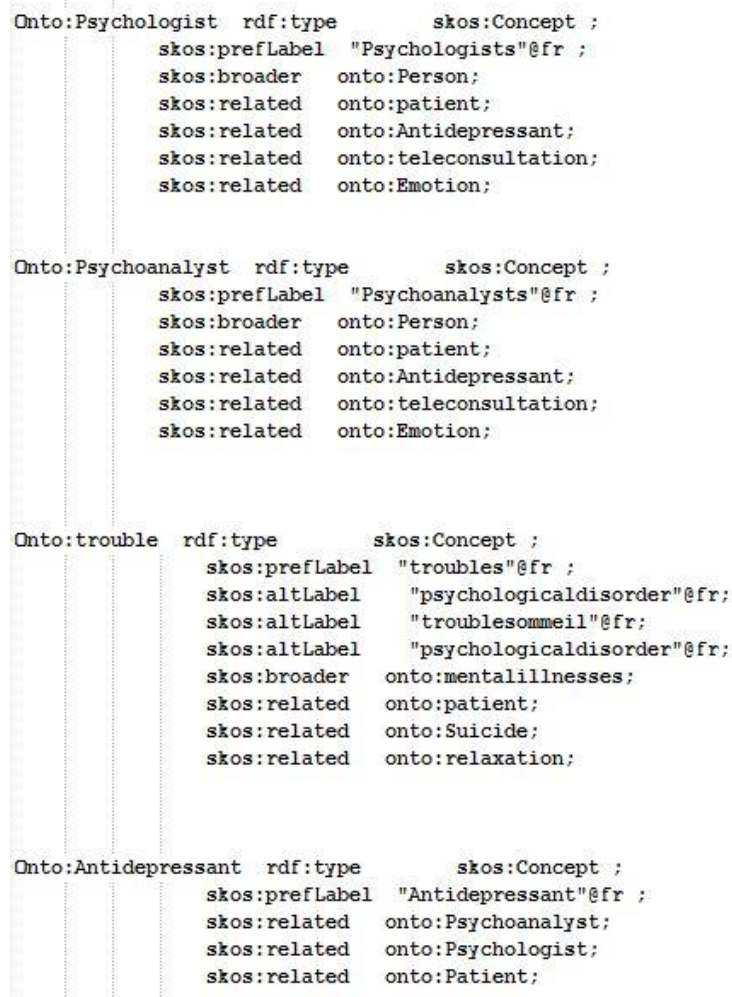

Figure. 6 Presentation of ontology 'OntoPsy-COVID-19'

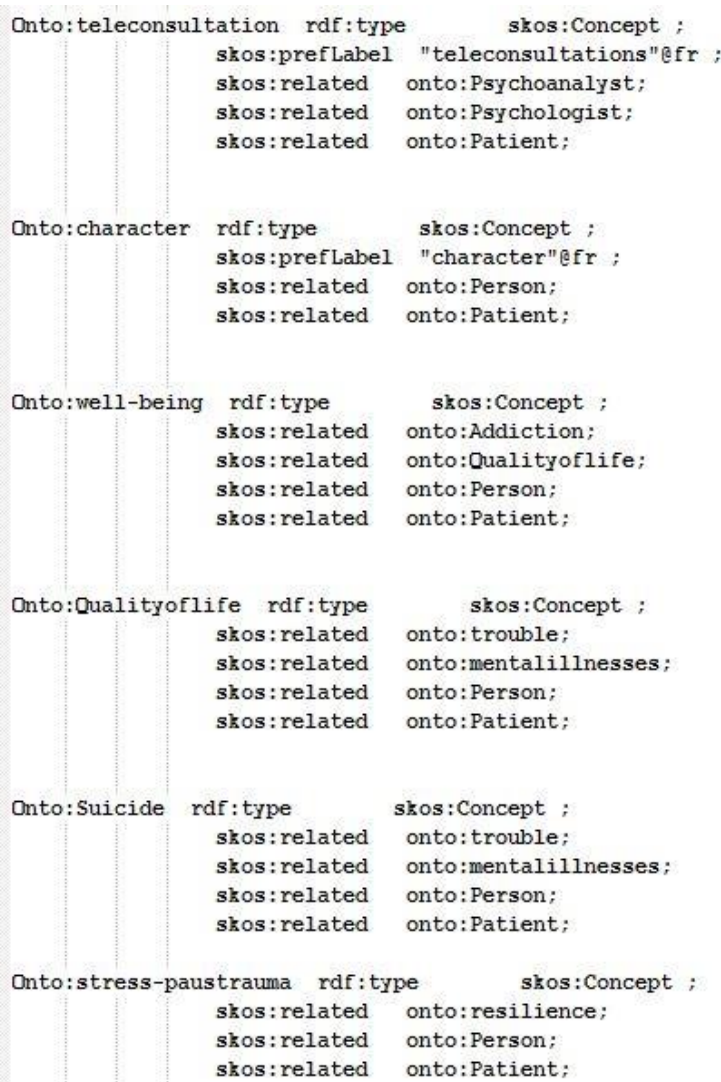

Figure. 7 Presentation of ontology 'OntoPsy-COVID-19'

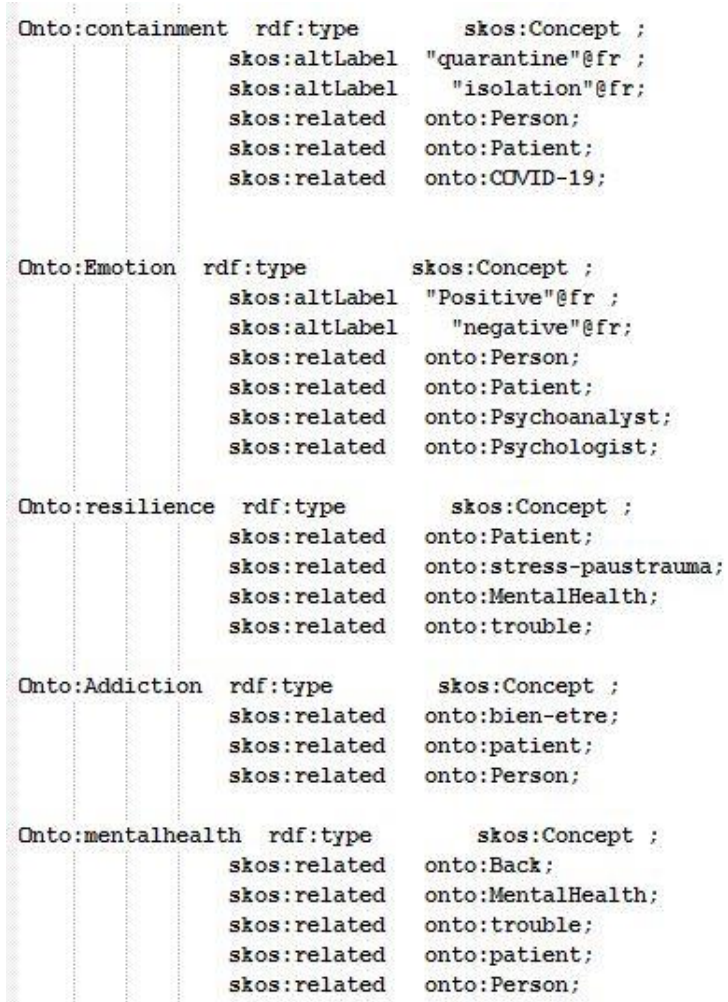

Figure. 8 Presentation of ontology 'OntoPsy-COVID-19'
Figure. 9 Presentation of ontology 'OntoPsy-COVID$19^{\prime}$

The objective is to validate the following points: The ontology adequately covers the knowledge of the domain. It takes into account the diversity of users and their professional level. The ontology provides portal users with quick and easy access to documents that meet their information needs.

Discussed the limitations encountered in this study and the mechanisms used to reduce its impact. Unfortunately, due to a lack of time and resources, we cannot overcome them: - Basic text used: the text is the starting point of our work, however, we have to be careful with some restrictions: To collect terms used by professionals in the field of psychology, we use a database of Google Texts and Google Scholar 


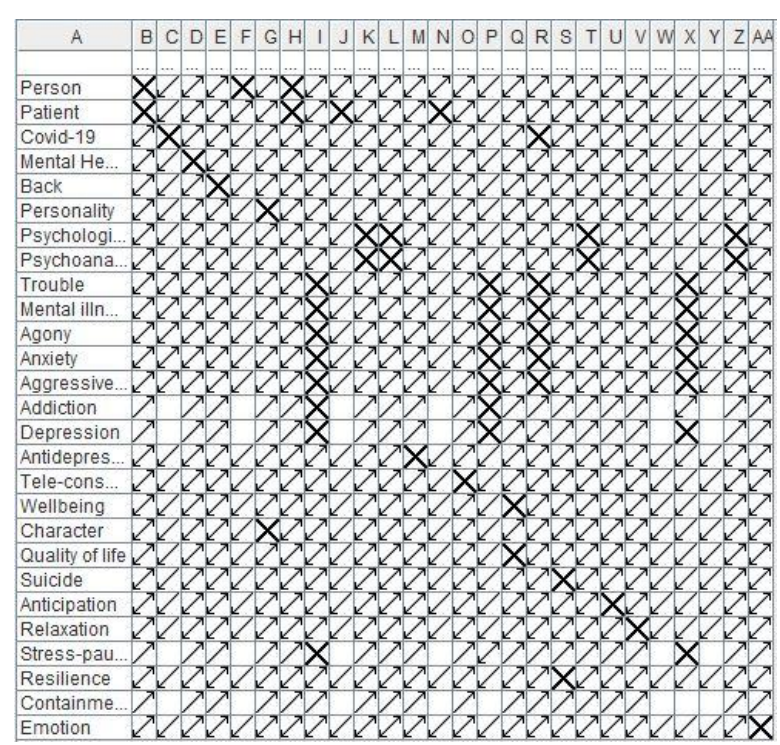

Figure. 10 Relations between the concepts of proposed ontology

articles published in 2020. Therefore, the authors of the articles we target are professionals in the field. The selection of profiles is based on the importance of their knowledge. - Text size varies and it seems difficult to give an accurate indication of the optimal number of words. In general, the text of articles related to the COVID-19 pandemic exceeds the text of the psychological literature.

The originality of our ontology lies in its idea of combining extracted term-candidates with the concordance and disambiguate with the method based on semantic similarity and ACF. This is in contrast to work that does not rely on a combined and structured method to guide the user in the construction of the ontology [23].

The value of our work is that the proposed approach has therefore made it possible to construct a psychological domain ontology in English relating to the state of people's mental health during the Fig. 10 presents a projection of relations between the concepts. COVID-19 pandemic, containing 24. These concepts are structured via 702 transversal relationships.

\section{Conclusion}

Throughout this article, we try to develop a domain ontology called "OntoPsy-COVID-19" through IR and terminology research. We begin by studying the salient elements of the text that form the basis of the text, which can be used to construct the ontology, based on the two hypotheses we specified at the beginning. Subsequently, we used methods adapted to our research to extract candidate words, remove ambiguity and define possible connections between them.
The motivation for developing this ontology was the lack of sufficient research in the sense of domain ontology, which modeled concepts related to the mental health of individuals during the COVID-19 pandemic. Therefore, our ontology fills this gap and describes the formal knowledge related to this domain ontology.

The purpose of this article is to describe the knowledge through the proposed ontology and using the SKOS language. Following this work, we intend to query this data and manipulate it through the SPARQL language, which uses graph patterns to determine the triplets that meet the conditions of the request, and which will be manipulated by the JEE language for the operations. The data is provided by SKOS and will be used as a platform to provide people with psychological support during the COVID-19 pandemic.

\section{Conflicts of Interest}

The authors declare that we do not have any circumstances or interest that may affect the results discussed in this manuscript.

\section{Author Contributions}

The paper conceptualization, methodology, software, and validation, have been done by $1^{\text {st }}$ author. Formal analysis, investigation, resources, data curation, writing - original draft preparation, writing - review, editing, and visualization, have been done by $1^{\text {st }}$ and $2^{\text {nd }}$ authors. The supervision, and project administration, has been done by $3^{\text {thd }}$ and $4^{\text {thd }}$ author.

\section{Acknowledgments}

This work is supported and financed by the University Hassan II of Casablanca- Morocco and The National Center for Scientific and Technical Research (CNRST) under the program "support program for scientific and technological research related to covid-19", project "Elaboration of a psychological and pedagogical support platform".

\section{References}

[1] F. Sarwar, S. Panatik, and F. Sarwar, "Psychology of Preventive Behavior for COVID19 outbreak". Journal of Research in Psychology, vol. 2, No.1, pp. 1-3, 2020.

[2] A. Pillay, B. Barnes, "Psychology and COVID19: impacts, themes and way forward". South African Journal of Psychology, vol. 50, No. 2, pp. 148-153, 2020. 
[3] S. Reddy, R. Sewpaul, Ronel, M. Mabaso, S. Parker, I. Naidoo, S. Jooste, T. Mokhele, S. Sifunda, and K. Zuma. "South Africans' understanding of and response to the COVID-19 outbreak: An online survey", Available at SSRN 3576939, 2020.

[4] M. Orkin, B. Roberts, N. Bohler-Muller, and K. Alexander. "The hidden struggle: The mental health effects of the Covid-19 lockdown in South Africa”, Daily Maverick, Vol. 13, 2020.

[5] G. Personeni. "Contribution of domain ontology for knowledge extraction from biomedical data", PhD thesis, University of Lorraine, N.Y, 2018.

[6] K. Drame, "Contribution to the construction of ontology and to the search for information: application to the medical field", University of Bordeaux, French, N.Y. 2014.

[7] A. Maedche, S. Staab, N. Stojanovic, R. Studer, and Y. Sure. "Semantic portal-The SEAL approach", Spinning the Semantic Web, pp.317359, 2003.

[8] D. Widdows, S. Peters, S. Cederberg, C. Chan. "Unsupervised monolingual and bilingual wordsense disambiguation of medical documents using UMLS", In: Proc. of the ACL 2003 workshop on natural language processing in biomedicine, pp. 9-16, 2003.

[9] P. Cuxac, A. Collignon, S. Gregorio, F. Parmentier, "From Massive Databases to the Web of Data: Disambiguation and Alignment of Geographic Features in Scientific Texts", In: Proc. of 12th International Colloquium of ISKO-France: Open data and mega-data in SHS: new challenges for the state and organization of knowledge, Montpellier, France, 2019.

[10] R. Donald, L. SOLDATOVA, "Are the Current Ontologies used in Biology Good Ontologies?", Nature Biotechnology, Vol. 23, No. 4, pp. 10951098, 2005.

[11] D. Harman, "Information retrieval: the early years", Foundations and Trends ${ }^{\circledR}$ in Information Retrieval, Vol. 13, No. 5, pp. 425577, 2019.

[12] H. Wache, T. Vogele, U. Visser, H. Stuckenschmidt, G. Schuster, H. Neumann, and S. Hubner. "Ontology-based integration of information- a survey of existing approaches", Ois@ijcai, 2001.

[13] R. Messai, " Ontology and Patient Services : Application to query reformulation", Computer Science and Language [cs.CL], University Joseph-Fourier - Grenoble I, French, 2009.

[14] T. M. Bridget and T. Pedersen, "Evaluating measures of semantic similarity and relatedness to disambiguate terms in biomedical text", Journal of Biomedical Informatics, Vol. 46, No. 6, pp. 1116-1124, 2013.

[15] H. Lin and J. Davis, "Computational and crowdsourcing methods for extracting ontological structure from folksonomy", In: Proc. of Extended Semantic Web Conference. Springer, Berlin, Heidelberg, pp. 472-477, 2010.

[16] B. Bachimont, "Semantic Engagement and Ontological Engagement: Design and Implementation of Ontology in Knowledge Engineering", Chapter Knowledge Engineering, chapter 19, pp. 305-323. 2000.

[17] M. T. Dhouib, C. Fron, and A. Tettamanzi, "Injection of Knowledge in a Sourcing Recommender System", In: Proc. of Web Intelligence. 2020.

[18] N. Niemants, "From recordings to corpora: transcription and extraction of interpretation data in the medical field", Meta: Translators' Journal, Vol. 63, No. 3, pp. 665-694, 2018.

[19] J. Vivaldi and H. Rodríguez, "Improving term extraction by system combination using boosting", In: Proc. of European Conference on Machine Learning, pp. 515-526, Springer, Berlin, Heidelberg, 2001

[20] P. Resnik, "Using information content to evaluate semantic similarity in a taxonomy", In: Proceedings of the 14th International Joint Conference on Artificial Intelligence, Montreal, 1995.

[21] D. Charlet and G. Damnati, "Simbow: a measure of semantic similarity metric between texts", In: Proc. of the 24th Conference on Automatic Natural Language Processing, pp. 126-133, 2017.

[22] D. Reineke and L. Romary, "Reference SKOS and TBX vocabularies used for comparing the two standards", hal-01883377v1, 2018.

[23] H. Azibi, N. Meddouri, and M. Maddouri, "Categorization of classification methods based on the Analysis of Formal Concepts", In: Proc. of 31th French-speaking days of Knowledge Engineering collection. 2020.

[24] V. B. V. N. Prasad, T. Ramarao, T. Rao, T. N. Rao, and K. Prasad, "Some Basic Principles on Posets, Hasse Diagrams and Lattices", Test Engineering and Management, Vol. 83, No. 2, pp. 10771-10775, 2020.

[25] I. Bunhas and Y. Slimani, "A hierarchical approach for semi-structured document indexing and terminology extraction", In: Proc. of International Conference on Information Retrieval \& Knowledge Management (CAMP), 2010. 
[26] W. P. R. C. Bere, G. Camara, S. Malo, S. Despres, M. Lo, and S. Ouaro, "Extraction of Relevant Data from Social Media Based on Termino-Ontological Resources: Application to Meningitis Surveillance via Twitter". In: Proc. of International Conference on Innovations and Interdisciplinary Solutions for Underserved Areas, Springer, Cham, pp. 52-63, 2020.

[27] P. D. Lunn, C. A. Belton, C. Lavin, F. P. McGowan, S. Timmons, and D. A. Robertson, "Using Behavioural Science to help fight the Coronavirus", Journal of Behavioral Public Administration, Vol. 3, No. 1, 2020.

[28] B. Dutta and M. DeBellis, "CODO: an ontology for collection and analysis of COVID-19 data", arXiv preprint arXiv: 2009.01210, 2020.

[29] Y. He, H. Yu, E. Ong, Y. Wang, Y. Liu, A. Huffman, H. Huang, J. Beverley, J. Hur, X. Yang, L. Chen, G. S. Omenn, B. Athey, and B. Smith, "CIDO, a community-based ontology for coronavirus disease knowledge and data integration, sharing, and analysis", Scientific Data, Vol. 7, No. 1, pp.1-5, 2020.

[30] M. F. Lopez, A. Gomez-Perez, J.P. Sierra, and A. P. Sierra, "Building a chemical ontology using methontology and the ontology design environment", IEEE Intelligent Systems and their Applications, Vol. 14, No 1, pp. 37-46, 1999.

[31] M. Uschold and M. King, "Towards a Methodology for Building Ontologies", Edinburgh : Artificial Intelligence Applications Institute, University of Edinburgh, 1995.

[32] M. Uschold and M. Gruninger, "Ontologies: Principles, methods and applications", Technical Report University of Edinburgh Artificial Intelligence Applications Institute AIAI TR, pp. 93-136, 1996.

[33] Y. Sure, S. Staab, and R. Studer, "On-toknowledge methodology (OTKM)", Handbook on Ontologies. Springer, Berlin, Heidelberg, pp. 117-132, 2004.

[34] A. Zouaq and R. Nkambou, "A survey of domain ontology engineering: Methods and tools", In: Proc. of Advances in intelligent tutoring systems, Springer, Berlin, Heidelberg, pp. 103-119, 2010.

[35] E. Simperl, "Reusing ontologies on the Semantic Web: A feasibility study", Data \& Knowledge Engineering, Vol. 68, No. 10, pp. 905-925, 2009.

[36] M. Hepp, J. Bruijn, "GenTax: A generic methodology for deriving OWL and RDF-S ontologies from hierarchical classifications, thesauri, and inconsistent taxonomies", In: Proc. of European semantic web conference. Springer, Berlin, Heidelberg, pp. 129-144, 2007. 